\title{
La nochebuena (Euphorbia pulcherrima), el tesoro mexica
}

Poinsettia (Euphorbia pulcherrima), a Mexica treasure

Victoria Arisai Aguilar Islas aryaguilar1401@gmail.com

https://orcid.org/0000-0002-4020-2446

Idalid Herrera González

idalidhgonzalez0523@gmail.com

https://orcid.org/0000-0002-9299-4430

Griselda Jácome Cervantes

grissjacome@gmail.com

https://orcid.org/0000-0002-2163-7209

Universidad Autónoma del Estado de Hidalgo

Recibido: 9 de agosto de 2021

Aceptado: 17 de septiembre de 2021

Publicado: 5 de enero de 2022

\section{Resumen}

En culturas prehispánicas, coloniales y actuales se ha utilizado la nochebuena, flor de pascua, cuetlaxochitl, sijoyo, poinsettia, entre otros nombres con que se conoce esta planta en México y Centroamérica. Su nombre científico es Euphorbia pulcherrima Willd. ex Klotzsch y es nativa de México y Guatemala. Presenta una característica que la hace distintiva de otras: tiene brácteas coloreadas (conjunto de hojas de colores que brotan a un mismo nivel). En este artículo mencionaremos características de la nochebuena, así como algunos usos comunes, tanto comerciales como medicinales, que se le dan a esta planta. También se mencionan los estados de la República Mexicana que más la producen, ya que forma parte de nuestra biocultura. La nochebuena representa una de las plantas ornamentales más icónicas, una contribución de México a la horticultura del mundo.

Palabras clave: plantas medicinales, uso comercial, plantas mexicanas.

\section{Abstract}

The poinsettia - also known as nochebuena (Christmas Eve flower), Easter flower, cuetlaxochitl, and sijoyo, among other names - has been used in pre-Hispanic, Colonial and current cultures. Its scientific name is Euphorbia pulcherrima Willd. ex Klotzsch and it is native to Mexico and Guatemala. It has a distinctive characteristic; namely, it has colored bracts (a set of colored leaves that all sprout from the same level). In this article we present the characteristics of the poinsettia and some common uses, both commercial and medicinal, that are given to this plant. We also identify the Mexican states where the most poinsettia is grown, since it is part of our bioculture. The poinsettia is one of the most iconic ornamental plants, a contribution from Mexico to world horticulture.

Keywords: medicinal plants, commercial use, Mexican plants.

\section{¿Sabes cómo es la nochebuena?}

Es una planta perteneciente a la familia Euphorbiaceae. Se puede encontrar en forma silvestre, sembrada en patios y jardines o cultivada para su venta (Galindo et al., 2012). Aunque las vemos en invernaderos o en puestos comerciales mucho más pequeñas, con el paso del tiempo y los cuidados adecuados las plantas llegan a medir de 1 hasta i4 metros de alto! Presenta un tallo leñoso y hueco por donde circula una savia lechosa o látex. Ramas fuertes y cilíndricas. Sus hojas son ovaladas, de color verde oscuro de tamaño mediano (llegan a medir $16 \mathrm{~cm}$ de largo). Lo más notable son las inflorescencias 
cubiertas por brácteas rojas (hojas modificadas) que son confundidas con pétalos; las flores se encuentran en el centro de estas brácteas agrupadas en una inflorescencia llamada ciatio. Las flores femeninas son pequeñas y reducidas en la parte central del ciatio, están rodeadas por flores masculinas, las cuales producen un estambre con anteras amarillas que contrastan con las brácteas rojizas (Sosa, 2021). Las brácteas tienen la tarea de atraer con colores llamativos (como rojo, amarillo, rosa, salmón) a los polinizadores, así como cuidar la inflorescencia, ya que esta se encuentra en el centro de la planta. Llegan a producir semillas que son muy pequeñas (miden 8 $\mathrm{mm}$ ) y tienen forma ovoide (CONABIO, 2010).

\section{De México para el mundo}

Las plantas de nochebuena en forma natural se distribuyen en la costa del Pacífico, desde Sinaloa en México, hasta Guatemala (Trejo et al., 2012). También se distribuyen en bosques tropicales secos de la cuenca del Balsas, particularmente en el norte de Guerrero y en Morelos. Se cultivan principalmente con fines comerciales en los estados de Morelos, Michoacán,
Ciudad de México, Puebla, Estado de México, Jalisco, Veracruz, Querétaro, Guanajuato, Chiapas y Guerrero (SADER, 2019).

La nochebuena simbolizaba para los mexicas la pureza y la nueva vida que obtenían los guerreros muertos en batalla. Existen algunas leyendas con respecto a su origen, una de ellas hace mención sobre una batalla en Taxco, en la que los mexicas derrotaron a los chontales. Alrededor de la población crecían hermosas flores blancas; sin embargo, después de la batalla las flores se marchitaron. Los habitantes decidieron cambiarle el nombre a "flores marchitas". Un año después, cuando los campos tenían que volver a crecer, los plantíos se cubrieron nuevamente de flores, pero ahora de color rojo debido a la sangre derramada (Enríquez, 2020).

En 1828 J. R. Poinsett, el primer embajador de los Estados Unidos en México, obtuvo nochebuenas de la zona de Taxco. Ese mismo año las mandó a un jardín botánico en Filadelfia. Para 1834, la flor se reproducía ya en Europa y gustó tanto que actualmente los descendientes de las plantas taxqueñas se cultivan en todo el mundo.

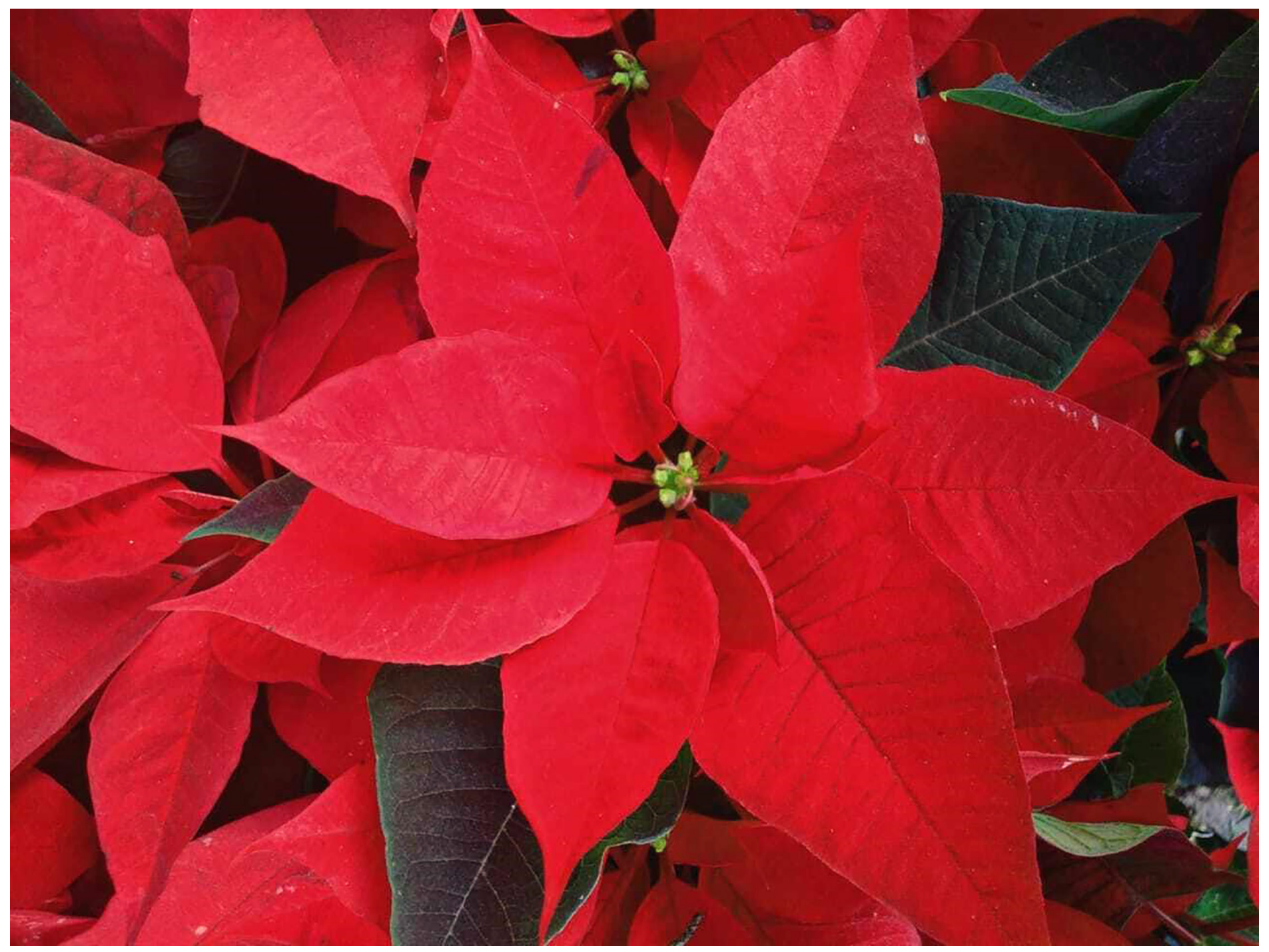

Euphorbia pulcherrima en el Mercado Benito Juárez, Pachuca de Soto Hidalgo. Fotografía: Idalid Herrera González. 


\section{Las nochebuenas en el México antiguo}

La nochebuena ha tenido una larga tradición hortícola que se remonta a los tiempos del imperio azteca. Su nombre científico, Euphorbia pulcherrima, significa en latín "la más bella". También es conocida como cuetlaxóchitl (en náhuatl) que significa "flor que se marchita" (Secretaría de Cultura, 2018). Los aztecas le daban usos prácticos a la planta, ya que extraían de las brácteas una tinta de color rojo púrpura para pintar sus telas que adquirían un color vistoso y el látex lo usaban para preparar una medicina que contrarrestaba la fiebre. El látex de la planta antiguamente también era utilizado como depilatorio. Los sacerdotes aztecas contemplaban esta flor antes de iniciar alguna ceremonia. También era el símbolo de la nueva vida, alcanzada por los guerreros muertos en la batalla. En la clasificación de plantas, creada por los médicos aztecas y recogida por el fraile Bernardino de Sahagún, se mencionan varias de sus propiedades: "la leche se utilizaba para curar infecciones cutáneas, heridas, hinchazones generadas por picadura de gusanos, afecciones respiratorias, anginas o tos. En las mujeres, la infusión de sus hojas, era utilizada para aumentar la cantidad de leche. Entre otras aplicaciones, era utilizada para fabricar tintura roja y utilizarla como cosmético, pigmento de pieles y algodón, así como para colorear códices y es tal su poder, que aún a la fecha se pueden apreciar en ellos dicha tonalidad" (Citado por Sans, 2017).

En el siglo XVII la flor fue incorporada por los frailes franciscanos de Taxco, Guerrero, a la celebración del nacimiento de Jesús, debido a sus colores brillantes y a que su época de floración coincide con dicha festividad (TrejoHernández et al., 2015).

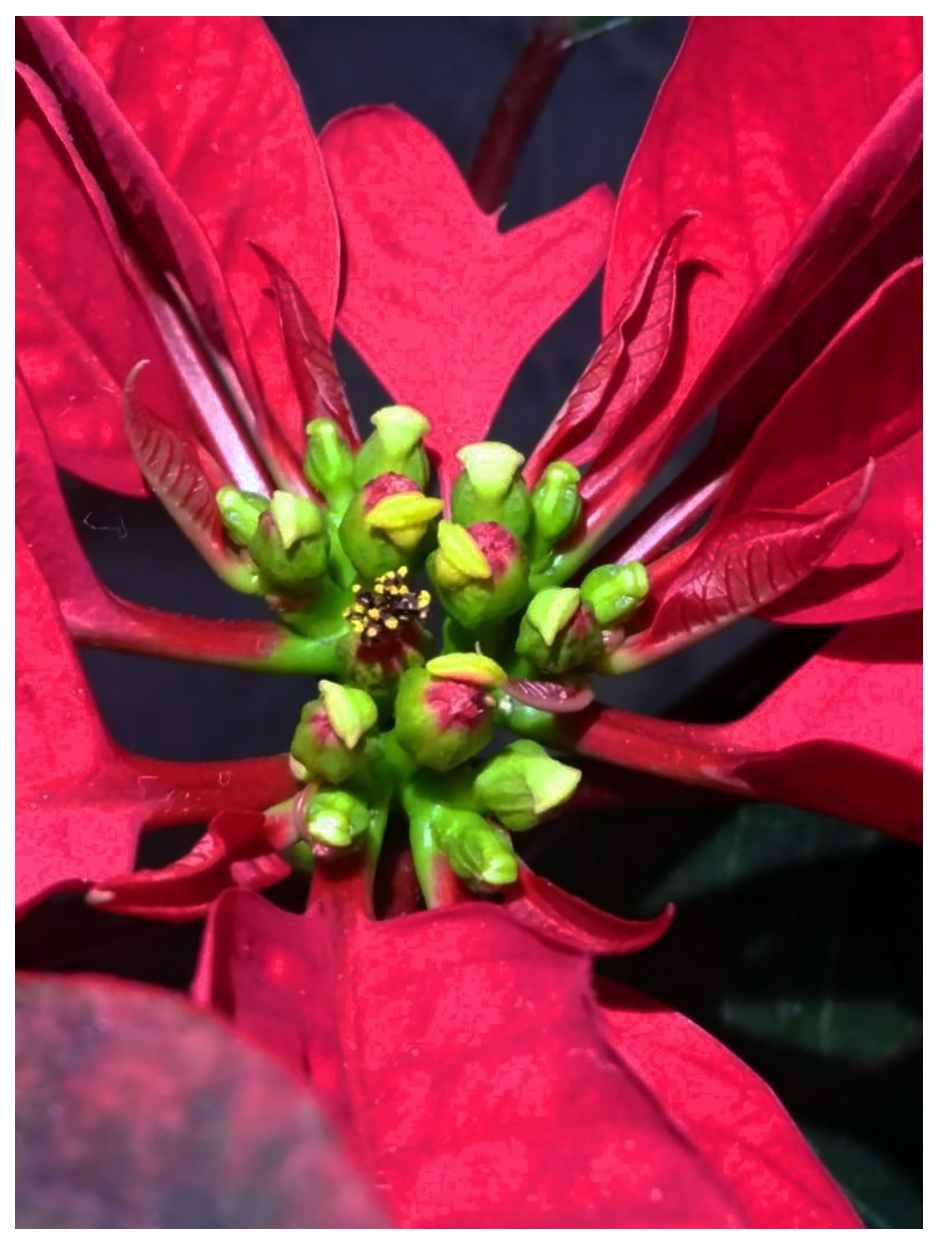

Ciatios de Euphorbia pulcherrima junto con sus glándulas. Nochebuena cultivada en un jardín de Pachuca de Soto, Hidalgo. Fotografía: Victoria Arisai Aguilar Islas.

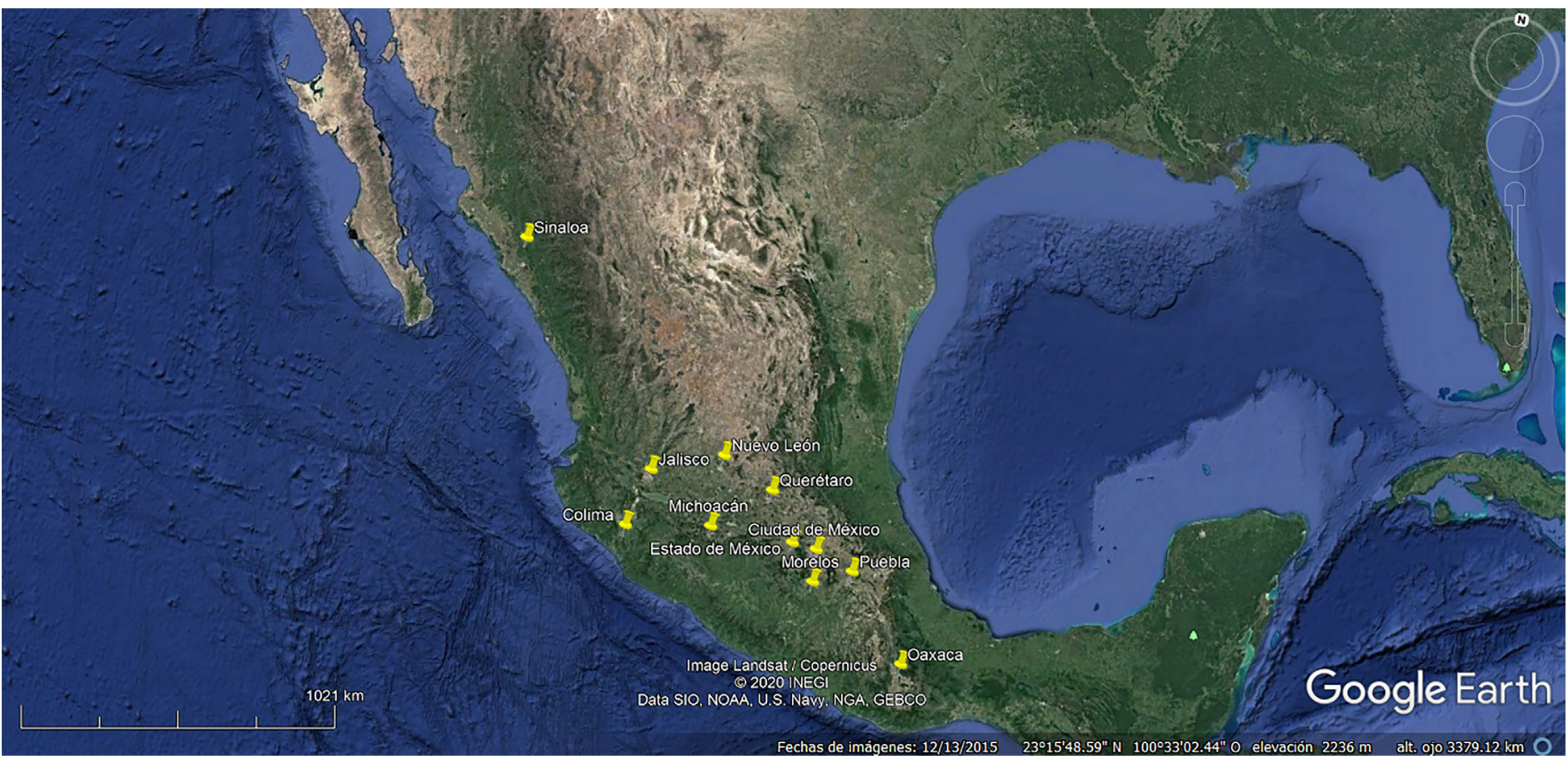




\section{Sus usos en la actualidad}

La nochebuena en el presente es utilizada principalmente como planta ornamental y se comercializa en macetas, preferentemente en la época decembrina (debido al color rojo que generalmente presentan sus brácteas); así, la podemos utilizar para el adorno de parques, jardines, edificios, corredores $\mathrm{u}$ ornamentales en casa. Las nochebuenas de la Ciudad de México, dada su historia, pueden ser germoplasma útil para el mejoramiento genético y la generación de nuevos cultivares mexicanos.

Su imagen ha sido utilizada como logotipo para tarjetas navideñas en la mayoría de los países cristianos, debido a un grupo de monjes franciscanos que iniciaron la tradición cuando con ella decoraron una peregrinación de la Fiesta del Santo Pesebre. Desde entonces, esta flor caracteriza y adorna las fiestas de esta época, no únicamente en México, sino en el resto del mundo.

\section{Navidad... iy luego?}

Las plantas de nochebuena son muy delicadas, por lo que tenerles un cuidado especial es lo adecuado para que luzcan hermosas por mucho más tiempo. Son plantas que necesitan una temperatura entre los $15^{\circ}$ y $25^{\circ} \mathrm{C}$, así como un lugar ventilado. También es recomendable colocarlas en un sitio en donde reciban la luz del sol, pero no directamente. Si las hojas se ponen amarillas o las ramas comienzan a doblarse, es posible que les falte luz o nitrógeno, pero lo más probable es que estén recibiendo demasiada agua. Algo muy importante para que las brácteas siempre se mantengan rojas o conserven su color si son de otra variedad, es no tallarlas con nada, no sacudirlas ni frotarlas con los dedos, esto puede hacer que se manchen y pierdan su color. Cuando las hojas empiezan a recobrar su color verde en febrero o marzo, es recomendable podar la planta para dejarla de aproximadamente veinte centímetros. En unas semanas tendrá muchos retoños que, a fin de año, engalanarán las fechas con sus colores llamativos.

\section{Agradecimientos}

A la Dra. Claudia Teresa Hornung Leoni por motivarnos y apoyarnos para la realización de este artículo en relación a lo aprendido en la materia Biología de Espermatofitas de la Licenciatura en Biología de la UAEH.

\section{Referencias}

CONABIO (Comisión Nacional para el Conocimiento y Uso de la Biodiversidad). 2010. Euphorbia pulcherrima-ficha informativa. Recuperado de: http://www.conabio.gob.mx/malezasdemexico/ euphorbiaceae/euphorbia-pulcherrima/fichas/ficha.htm

Enríquez, Y. 2020. La flor de Nochebuena: leyendas, cuidados y dónde comprarlas en México. Recuperado de: https://mexicorutamagica. $\mathrm{mx} / 2020 / 12 / 11 /$ nochebuena-leyenda-cuidados-dondecomprarla-en-mexico/

Galindo-García, D.V., Alia-Tejacal, I., Andrade-Rodríguez, M., ColinasLeón, M. T., Canul-Ku, J.y Sainz-Aispuro, M de J.2012. Producción de nochebuena de sol en Morelos. Revista Mexicana de Ciencias Agrícolas, 3: 751-753. Recuperado de: http://www.scielo.org.mx/ scielo.php?script=sci_arttext\&pid=S2007-09342012000400010

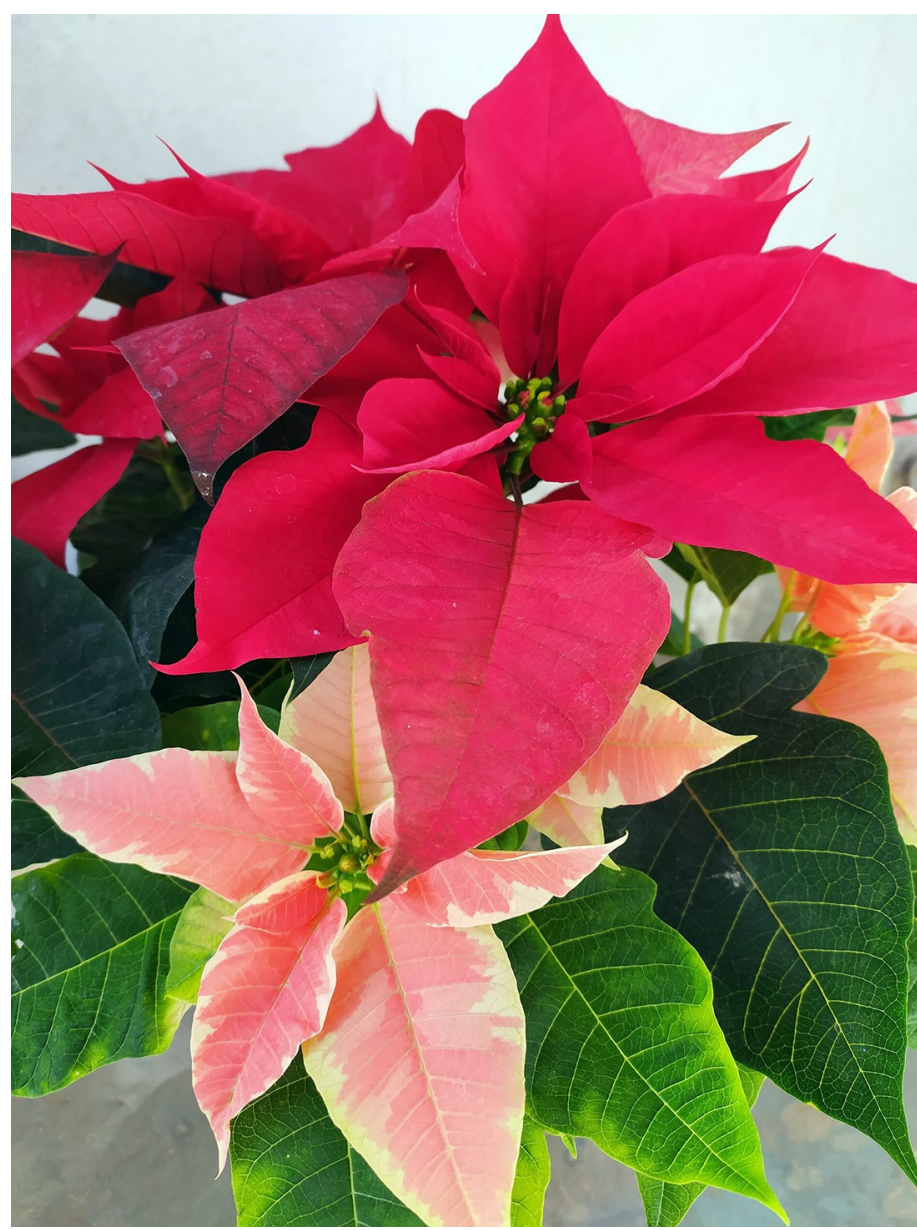

Distintos colores de Euphorbia pulcherrima, nochebuena cultivada en un jardín de Pachuquilla, Hidalgo. Fotografía: Griselda Jácome-Cervantes.

Sans, D. 2017. El Origen azteca de la flor de nochebuena y su historia Culturamas.es. Recuperado de: https://www.culturamas. es/2017/12/06/el-origen-azteca-de-la-flor-de-nochebuena-y-suhistoria/

SADER (Secretaría de Agricultura y Desarrollo Rural). 2019. Producción de nochebuena en la Ciudad de México. Recuperado de: https:// www.gob. $\mathrm{mx} /$ agricultura/cdmx/articulos/produccion-denochebuena-en-la-ciudad-de-mexico?idiom=es

Secretaría de Cultura. 2018. ¿Sabías que en náhuatl la flor de nochebuena (Cuetlaxóchitl) significa flor que se marchita? Recuperado de: https://www.gob.mx/cultura/es/articulos/flor-de-nochebuena185645 ? idiom $=$ es

SEMARNAT (Secretaría de Medio Ambiente y Recursos Naturales). 2010. Plantas medicinales de la farmacia viviente del CEFOFOR: usos terapéuticos tradicionales y dosificación. Recuperado de: http://www.conafor.gob.mx/biblioteca/Plantas_medicinales_de_ la_farmacia_viviente-Conafor.pdf

Sosa, V. 2021. La flor de Nochebuena. Recuperado de: http://www.inecol. $\mathrm{mx} /$ inecol/index.php/es/component/content/article/17-cienciahoy/856-la-flor-de-nochebuena

Trejo-Hernández. L., Olson-Zúñica. M. E. y Bye-Boettler., A. R. 2015 Datos históricos y diversidad genética de las nochebuenas (Euphorbia pulcherrima) del Distrito Federal, México. Revista Mexicana de Biodiversidad, 86: 478-485. Recuperado de: http://www.scielo.org.mx/pdf/rmbiodiv/v86n2/1870-3453rmbiodiv-86-02-00478.pdf

Trejo, L., Feria, T., Olsem, K., Eguiarte, L., Arroyo, B., Gruhn, J. y Olson, M. 2012. Poinsettia's wild ancestor in the Mexican dry tropics: historical, genetic, and environmental evidence. American Journal of Botany, 99 (7): 1146-1157. Recuperado de: https:// bsapubs.onlinelibrary.wiley.com/doi/epdf/10.3732/ajb.1200072 